\title{
Effect of exposure to an extremely low frequency-elec- tromagnetic field on the cellular collagen with respect to signaling pathways in osteoblast-like cells
}

\author{
Akira Soda', Toshitaka Ikehara ${ }^{1 *}$, Yohsuke Kinouchi ${ }^{2}$, and Kazuo Yoshizaki \\ ${ }^{1}$ Department of Physiology, Pathophysiological Preventive Medicine, Institute of Health Biosciences, \\ the University of Tokushima Graduate School ; and ${ }^{2}$ Life System, Institute of Technology and Sci- \\ ence, Graduate School, the University of Tokushima, Tokushima, Japan
}

\begin{abstract}
The effect of exposure to extremely low frequency-electromagnetic field (ELFEMF : $3 \mathrm{mT}, 60 \mathrm{~Hz}$ ) on differentiation of mouse osteoblast-like MC3T3-E1 cells was examined together with addition of insulin-like growth factor I (IGF-I). As a marker of the differentiation, the cellular collagen content was determined by the absorbance of Sirius red-stained cells measured at the wavelength of 510-520 $\mathrm{nm}$ with an imaging microspectroscopy.
\end{abstract}

Exposure to ELF-EMF increased significantly the collagen in the cells. Treatment with PD98059, an inhibitor of extracellular signal-regulated kinase 1/2 (ERK1/2) activation, reduced the collagen in all of the cells examined on control, IGF-I addition and ELFEMF exposure, however, PD98059 did not prevent the increase in the collagen caused by ELF-EMF exposure, and IGF-I also increased the collagen in the presence of the inhibitor. When phosphatidylinositol 3-kinase (PI3K) pathway was inhibited by LY294002, the increase in collagen induced by ELF-EMF exposure was accelerated, however, the increase in collagen observed by IGF - I addition was suppressed. Treatment with SB203580, an inhibitor of p38 mitogen - activated protein kinase (p38 MAPK), suppressed the increase in the collagen induced by ELF-EMF exposure, whereas IGF-I addition increased the collagen in the presence of the inhibitor.

These results suggested that collagen synthesis stimulated by ELF-EMF exposure was carried out by the participation of p38 MAPK pathway, and that PI3K pathway may have the role to suppress the collagen synthesis induced by ELF-EMF exposure, and that the suppression of the PI3K pathway may allow the acceleration of the collagen synthesis. J. Med. Invest. 55 : 267-278, August, 2008

Keywords : ELF-EMF, ERK1/2, PI3K, p38 MAPK, collagen, osteoblast, MC3T3-E1 cell

\section{INTRODUCTION}

Since Bassett CAL, et al. (1) have first applied

Received for publication April 14, 2008 ; accepted July 23, 2008.

*Present address : Yasuda Woman's University, Hiroshima 7310153, Japan

Address correspondence and reprint requests to Akira Soda, Department of Physiology, Pathophysiological Preventive Medicine, Institute of Health Biosciences, the University of Tokushima Graduate School, Kuramoto-cho, Tokushima 770-8503, Japan and Fax : +81-88-633-9443. electromagnetic pulses to bone fracture more than forty years ago, exposure to electromagnetic fields has been used clinically to enhance healing of bone fracture (2). The mechanisms of fracture healing have been extensively studied and the influence of magnetic field exposure on various biological functions has been reviewed (3). However, the basic mechanism of the acceleration of fracture healing has not been sufficiently elucidated. 
We have studied the effects of exposure to magnetic and electromagnetic fields (EMFs) on ion transports of cultured cells $(4,5)$, and found that exposure to strong static magnetic fields, $1 \mathrm{~T}$ or more, does not significantly influence cell functions (4), even when the magnetic fields are either nonhomogeneous with large gradients (6) or applied for seven months (7). In contrast, we have shown that exposure to strong time-varying EMFs inhibits intracellular $\mathrm{Ca}^{2+}$ release $(8,9)$ and $\mathrm{K}^{+}\left(\mathrm{Rb}^{+}\right)$influx (10) in cultured cells. Exposure to extremely low frequency-electromagnetic field (ELF-EMF) also influences the structure of cell membrane protein (11). These results suggest that the effect of magnetic field seems to be caused by eddy current induced in the culture medium rather than magnetic field itself, because the time-varying magnetic field but not the static magnetic field has the effect on the cells. Similarly, other investigators have suggested that the responses of mouse osteoblast-like MC3T3-E1 cells to ELF-EMF exposure depend on the induced electric field, based on a finding that cells oriented parallel to the induced electric field showed a decrease in cell length and an increase in roundness (12). Exposure to ELF-EMF has been also reported to induce the differentiation of human skin fibroblasts to fibrocytes in culture (13).

Mouse osteoblast-like MC3T3-E1 cells are model cells of bone formation in culture and express distinctly the proliferative and differentiating phases (14). During the initial phase of culture, cells proliferate exponentially in less than 10 days and thereafter they stop to multiply and enter the differentiation phase, characteristic of collagen synthesis, expression of alkaline phosphatase (ALP) activity, and extracellular calcification at the final stage. The differentiation is dependent upon the temporal regulation of multiple signaling pathways and the roles of mitogen-activated protein kinases (MAPKs), such as p38 MAPK, in bone forming cells have been studied (15).

It is well recognized that insulin-like growth factor I (IGF-I) plays a crucial role in the regulation of growth and bone metabolism, and extracellular signal-regulated kinase $1 / 2$ (ERK1/2, also called $\mathrm{p} 44 / \mathrm{p} 42$ MAPK) plays a role in the IGF-I stimulated activation of alkaline phosphatase in MC3T3$\mathrm{E} 1$ cells (16). IGF-I also enhances $\mathrm{G}_{1} / \mathrm{S}$ phase progression of the cell cycle in skeletal muscle satellite cells through the activation of phosphatidylinositol 3-kinase (PI3K) pathway (17), and regulates the growth of human intestinal muscle cells (18), and the proliferation and survival of human osteoblastic cells (19).

In this study, we examined the effect of ELFEMF exposure, together with the effect of IGF-I, on the differentiation of mouse osteoblast-like MC 3T3-E1 cells in culture. We adopted collagen synthesis as a target, because the synthesis starts at relatively early stage of the differentiation and it takes place possibly by participation of some signaling pathways. We intended to clarify whether the signaling pathways involving ERK1/2, PI3K and p38 MAPK were related to the effects on the collagen synthesis, using each inhibitor of the signaling pathways.

We first set up a system consisting of combined coils and a culture incubator to expose the cells under physiological conditions to sinusoidal ELFEMF with magnetic flux density even. Secondly, we analyzed the effect of ELF-EMF exposure on the intracellular collagen content together with IGF-I addition, using a technique of imaging microspectroscopy in combination with cell staining specific to discriminate collagen from other proteins.

\section{MATERIALS AND METHODS}

\section{Cell Culture}

We used an established cell line of osteoblastlike MC3T3-E1 cells derived originally from cranial bone of a newly born mouse (strain C57BL/6) (14). The cells had been maintained in a culture medium consisting of modified Eagle's minimum essential medium, $\alpha$-MEM (MP Biomedical, Inc., Eschwege, Germany), supplemented with $10 \%$ fetal bovine serum (FBS), ascorbic acid $(50 \mu \mathrm{g} / \mathrm{ml})$ and $\alpha$-glycerophosphoric acid disodium salt (5 $\mathrm{mM})$, and added with penicillin (100 units/ml) and streptomycin $(100 \mu \mathrm{g} / \mathrm{ml})$. They were inoculated in plastic culture dishes (35 or $60 \mathrm{~mm}$ diameter, Corning Glass Works, Corning, USA) and placed in an incubator in a humid atmosphere of $5 \% \mathrm{CO}_{2}$ and $95 \%$ air to keep medium $\mathrm{pH}$ at $7.2-7.4$ at $37^{\circ} \mathrm{C}$. The culture medium was changed once $3-4$ days. When the cell population became dense, the cells were dispersed in a medium containing $0.1 \%$ trypsin and $0.1 \%$ collagenase in the balanced salt solution (BSS : $135 \mathrm{mM} \mathrm{NaCl}, 5.6 \mathrm{mM} \mathrm{KCl}, 1.2 \mathrm{mM} \mathrm{CaCl}_{2}$, $10 \mathrm{mM}$ glucose and $20 \mathrm{mM}$ 4-(2-hydroxyethyl) -1piperazineethane-sulfonic acid (HEPES) adjusted at $\mathrm{pH} 7.4$ with $\mathrm{NaOH}$ ). For experiments, cells were inoculated in the plastic culture dishes $(60 \mathrm{~mm}$ di- 
ameter) at a density of $9 \times 10^{4}$ cells $/ \mathrm{ml}$, and were cultured for 11-21 days. Each culture period was stated in the Results.

\section{Chemicals}

Most reagents were of commercial reagent grade. IGF-I, LY294002 [2-(4- morpholinyl) -8-phenyl4H-1-benzopyran-4-1], SB203580 [4-(4-fluorophenyl) - 2- (4- methylsulfinylpheny) -5 - (4-pyridyl) 1H-imidazole], and Fast green were purchased from Wako Pure Chemical Indust. (Osaka) ; trypsin and collagenase from Difco Laboratories (Detroit USA) ; PD98059 [2'-amino-3'-methoxyflavone] from Calbiochem. Co. (USA and Canada) ; and Sirius red from Polysciences Inc. (Warrington, USA).

\section{Structure of Coils}

Magnetic field was produced by a coil wound around a cylindrical plastic (polyvinyl chloride) container $(37 \mathrm{~cm}$ outer diameter ; $34.5 \mathrm{~cm}$ inner diameter; $33 \mathrm{~cm}$ length) placed vertically (Figs. 1 and 2). The coil consisted of primary and secondary coils made of enameled wires $(2.12 \mathrm{~mm}$ diameter, electric resistance $5.66 \Omega / \mathrm{km}$ ). The primary coil was made of the wire wound 150 times around the plastic container. Theoretical calculation revealed that the magnetic flux density was about $2.3 \mathrm{mT}$ at the central axis of the primary coil when coil current was $1.94 \mathrm{~A}$ at a frequency of $60 \mathrm{~Hz}$. The magnetic flux density tended to increase as the distance from the central axis of the coil increased, approaching the coil wires. It was higher by $0.3 \mathrm{mT}$ at the periphery than at the center of the coil (Fig. $3 \mathrm{~A}$ : the primary coil).

We introduced the secondary coil to correct the uneven distribution of the magnetic flux density of the primary coil (Fig. 2A). The secondary coil was made of the same wire and separated into two pieces, each of which was made of three layers. The wire was wound 33 times every layer. The pieces of the secondary coil were overlaid on the primary coil at the upper and lower ends of the latter. The secondary coil produced the magnetic flux density of about $0.7 \mathrm{mT}$ at the center of the coil. As the coil was of Helmholtz type, the magnetic flux density was the highest at the central axis and gradually decreased as the distance from the axis increased. The distribution of the magnetic flux density made a contrast to that of the primary coil. Such contrastive changes in the magnetic flux density produced by the two coils were offset inside the combined coils, resulting in the even distribution of magnetic flux density of about $3.0 \mathrm{mT}$, regardless of the distance (Fig. 3A). We also calculated the magnetic flux density at different heights in the combined coils. There were no significant differences in the magnetic flux density at different heights within at least $4 \mathrm{~cm}$ from the middle of the combined coils, where the cells in culture were placed (Fig. 3B).

\section{Incubator placed in Coils}

Plastic incubators $(25 \mathrm{~cm}$ length, $20 \mathrm{~cm}$ width and $10 \mathrm{~cm}$ height) were designed to maintain culture conditions physiological (Fig. 2B). About 12 culture dishes (60 $\mathrm{mm}$ diameter) were set in three

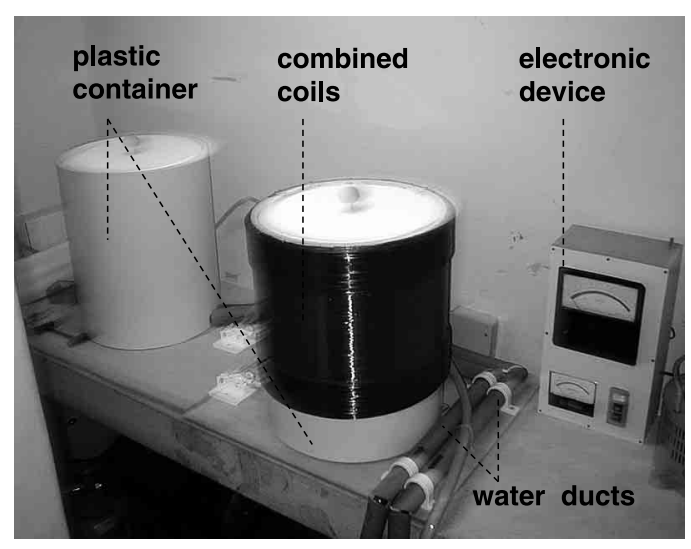

Fig. 1 A couple of plastic container devices for exposing a group of cultured cells to an extremely low frequency-electromagnetic field (ELF-EMF) produced by the coils and for placing another group of cells outside the ELF-EMF. An incubator containing culture dishes was placed in the middle of each plastic container (see Fig. 2B).

A
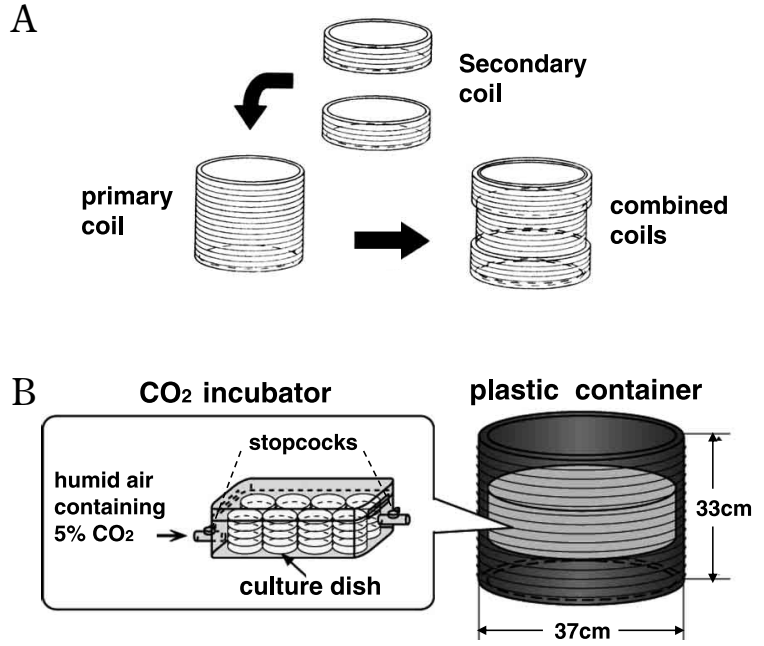

Fig. 2 A: Structure of combined coils consisting of primary and secondary coils. B : An incubator for keeping cells cultured under physiological conditions while they were exposed to ELF-EMF. 
A

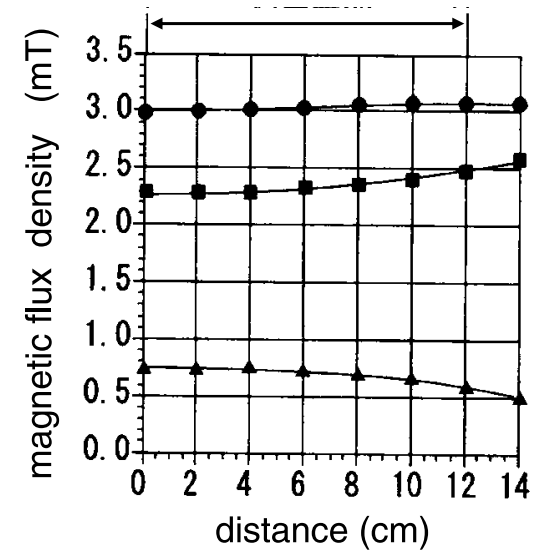

B
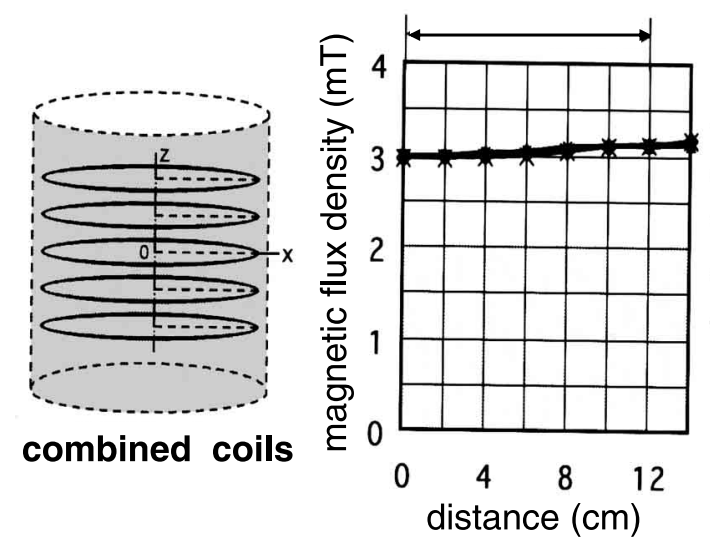

Fig. 3 A: Distribution of the magnetic flux density on a horizontal plane as a function of the distance (x) from the central axis of each coil. $\square$, the primary coil ; $\boldsymbol{\Lambda}$, the secondary coil ; , the combined coils. B : Distribution of the magnetic flux density on the various heights $(z)$ of horizontal planes, as a function of the distance (x) from the central axis of the combined coils. $4 \mathrm{~cm}$ above the middle of the coils (i.e., $z=4 \mathrm{~cm}$ ); $\boldsymbol{O}, 2 \mathrm{~cm}$ above that ; $\mathbf{\Delta}, 0 \mathrm{~cm}$ on the middle of the coils ; $\times, 2$ cm below that; $*, 4 \mathrm{~cm}$ below that; $\longleftrightarrow$, the range from the central axis, in which culture dishes were placed.

layers in the incubator. Air in the incubator containing culture dishes was replaced with humid gas consisting of $5 \% \mathrm{CO}_{2}$ and $95 \%$ air by passing the gas for a minute from a plastic tube connected to one side of the incubator to the similar tube connected to the other side. The incubator became airtight when stopcocks connected to the tubes were closed. It was placed in the middle of the plastic container inside the combined coils for exposure to extremely low frequency-electromagnetic field (ELF-EMF). The incubator was sandwiched by two cylindrical water baths, one of which was set to keep contact with the lid and the other one with the bottom of the incubator. Water in the baths was continuously circulated by a pump equipped with a heater (Coolnics Circulator CTE82A, Yamato Science Co. Ltd., Tokyo), so that temperature inside the incubator was kept constant at $37 \pm 0.2^{\circ} \mathrm{C}$ as measured with a thermometer (Takara D117, Takara Indust. Co., Tokyo). Another incubator, placed outside the ELF-EMF in a similar plastic container covered with a layer of plastic foam (about $2.5 \mathrm{~cm}$ thickness), was used for control (Fig. 1).

\section{Induction Currents in Culture Medium}

Since the culture medium in a culture dish is electrically regarded as a disc conductor, the changes in the magnetic flux passing vertically through the disc must cause circular induction currents in the medium. Let the effective induction current density be $i_{e}\left[\mathrm{~A} / \mathrm{m}^{2}\right]$, the conductivity of the culture medium $\sigma[\mathrm{S} / \mathrm{m}]$, the effective magnetic flux density $B_{e}[\mathrm{~T}]$, frequency of the ELF-EMF $f[\mathrm{~Hz}]$, and the distance from the center of the disc $r$ [m]. Then, the following equation can be derived from Maxwell's equation and Ohm's law :

$$
i_{e}=\pi r \sigma f B_{e} \text {. }
$$

Equation [1] indicates that the density of currents induced in the culture medium is proportional to $r$. We determined $\sigma$ of $\alpha$-MEM to be 1.59 $\mathrm{S} / \mathrm{m}$ at $37.2^{\circ} \mathrm{C}$ and the value was almost unchanged in the $\mathrm{pH}$ range from 5.73 to 8.98 . Using equation [1], the values of $\sigma$ and $f(60 \mathrm{~Hz})$, we calculated that the density of induced current is zero at the center of the culture dish (60 mm diameter) and about $2.7 \mu \mathrm{A} / \mathrm{cm}^{2}$ at the periphery.

\section{Exposure of Cells to ELF-EMF and Addition of IGF-I and Inhibitors}

Cells were exposed to the ELF-EMF $(60 \mathrm{~Hz}, 3$ $\mathrm{mT}$ ) produced by the combined coils, and/or were treated with IGF-I $(200 \mathrm{ng} / \mathrm{ml})(16,20)$ in the presence or absence of an inhibitor $(10 \mu \mathrm{M})$ such as LY294002 (18), SB203580 (15, 21), and PD 98059 (21), respectively, for the last 3 days of every culture period shown in the Results.

\section{Determination of Intracellular Collagen Content}

At the end of ELF-EMF exposure, cells were fixed with $4 \%$ paraformaldehyde. Fixed cells were stained with $0.1 \%$ Sirius red dissolved in a phosphate buffer solution, and when necessary with $0.1 \%$ Fast green, for 30 min on a shaker and then washed with distilled water several times (22). The stained sample was covered with liquid paraffin to avoid drying. Collagen in the cells was stained specifically with Sirius red, and non-collagen proteins with Fast green, respectively. Figure 4 shows absorption spectra of diluted solutions of Sirius red and Fast green in the wavelength range from 450 


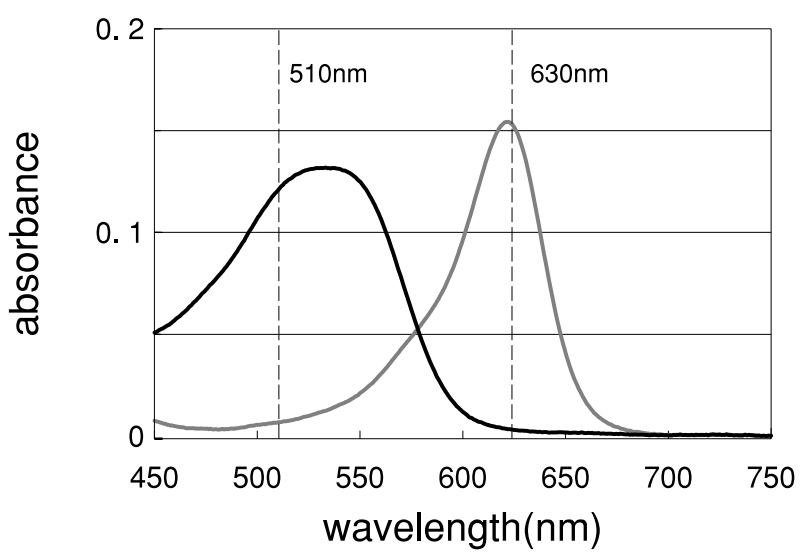

Fig. 4 Absorption spectra of Sirius red (black curve) and Fast green (gray curve). The diluted solutions of the pigments were $0.0005 \%$ Sirius red and $0.0001 \%$ Fast green. Wavelengths used for determinations of collagen and non-collagen protein were $510-520 \mathrm{~nm}$ and $630-640 \mathrm{~m}$, respectively.

to $750 \mathrm{~nm}$. The absorption spectra of Sirius red and Fast green were the maxima at around 530 and 620 $\mathrm{nm}$, respectively. To estimate the collagen content from the absorbance of Sirius red, we used the wave length at 510-520 nm to avoid the contribution of Fast green. Non-collagen proteins was not determined in this study.

We used an imaging microspectroscopy to quantify the cellular contents of collagen. Spectral information of stained cells was recorded with a CCD camera built in a multispectral imaging photometer (ImSpector, Spectral Imaging Ltd., Oulu, Finland). Namely, the observed light coming straightly through a sample dispersed with a transmission grating in a wavelength range of $380 \sim 780 \mathrm{~nm}$ at a wavelength resolution of $2 \mathrm{~nm}$. The spectral image was captured with the CCD camera and recorded on a video frame. The CCD camera was loaded with an image intensifier (luminescence gain 25,000, sensitivity $20 \mu \mathrm{lx}$, wavelength range $380 \sim 850 \mathrm{~nm}$, wavelength resolution $2 \mathrm{~nm}$ ). The stage of an inverted microscope (IX70, Olympus Co. Ltd, Tokyo) was moved to a single direction with a computer-controlled step motor, and the measurement times were 0.1-2.1 sec/line and $30 \mathrm{sec} /$ frame. For the data analysis and image display, we used a computer software (PSAM-700, Kawatetsu Technoresearch Co. Ltd., Tokyo). ImSpector system had an advantage of obtaining many pieces of information on spectra and positions of multiple points in an image. This was in contrast to traditional microspectrophotometry, which obtains spectral information from only a single point in an image. In addition, it was possible to display spectrum at any point on the image and differential spectrum between two points at the same time. Display of the absorbance at a specific wavelength was also possible.

In this study the single point analysis method was used to examine the localization effect, as shown later in Fig. 5. We also used the spectral image analysis method, i.e., estimation of the collagen content from the absorbance image at the wavelength $510-520 \mathrm{~nm}$ in the same video frame as in a figure shown later in Fig. 9. The quantification of the absorbance image was repeatedly on several places in the periphery of a culture dish to analyze the relative changes in the collagen contents, as shown later in Figs. 6, 7 and 8.

\section{Statistic Analysis and Cell Sampling}

Since cells belonged to the same population, significance of the difference on means of the absorbance between two experimental groups was checked by ' $t$-test'. The difference was regarded as significant at $\mathrm{p}<0.05$.

\section{RESULTS}

Equation [1] indicates that the density of eddy currents induced in the culture medium by ELFEMF is proportional to the distance from the center of culture dish. Therefore, we compared the effects of ELF-EMF exposure on the absorbance of Sirius red between cells located at around the center and cells at the periphery of the dishes, and the spectral analysis on a single point in the image were repeated on two culture dishes to examine the localization effect. On ELF-EMF exposure the absorbance of Sirius red at the periphery was significantly larger than that at around the center in the cells cultured for 14 days (Fig. 5A). The similar tendency was observed in cells exposed to ELFEMF in the presence of IGF-I, although the difference was statistically not significant. No significant difference of the absorbance between the cells located at the two positions was observed in both control and IGF-I groups.

We further examined the difference of absorbance, using cells cultured for 21 days (Fig. 5B). These cells contained larger amount of collagen than the cells cultured for 14 days, and marked increase in the absorbance was observed in the cells exposed to ELF-EMF, however, there was no significant difference with respect to the cell location. The enhancing effect of ELF-EMF exposure on 

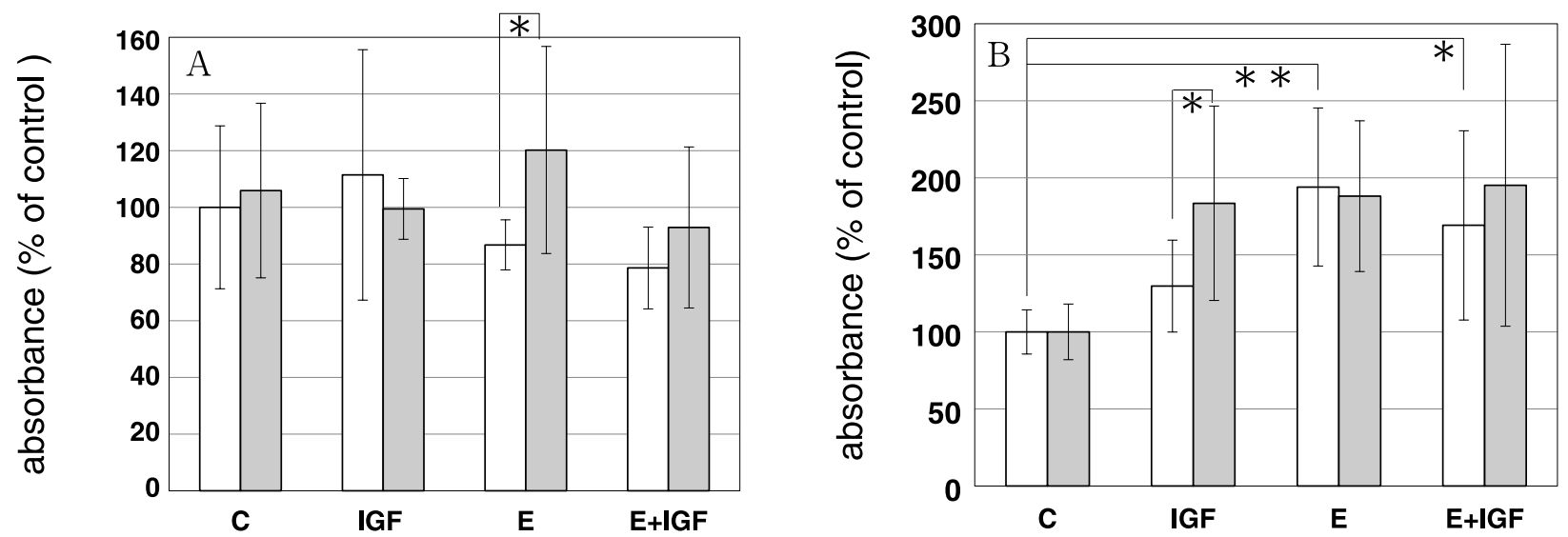

Fig. 5 The effect of ELF-EMF exposure on the absorbance of MC3T3-E1 cells stained with Sirius red, and the localization effect examined at around the center (white columns) and at the periphery (gray columns) in the culture dishes. Cells were exposed to ELF-EMF $(60 \mathrm{~Hz}, 3 \mathrm{mT})$, in the presence or absence of IGF $(200 \mathrm{ng} / \mathrm{ml})$, for the last 3 days in the culture periods. A : Cells cultured for 14 days. B : Cells cultured for 21 days. C, control ; IGF, IGF-I addition ; E, ELF-EMF exposure ; and E+IGF, ELF-EMF exposure with IGF-I addition. Height and bar of each column represent mean and standard deviation of the absorbance. In each of two culture dishes every group 10 different points were sampled at random for analysis. The symbols * and ** represent significantly different at $\mathrm{p}<0.05$ and $\mathrm{p}<0.01$, respectively.

cells seemed to be maximum even at around the center, making localization-dependent discrimination difficult. When cells were treated with IGF-I, the absorbance at the periphery was unexpectedly larger than that at around the center. Further study is necessary to elucidate the reason.

In the following studies we analyzed only the periphery cells in the culture dish, and the collagen content was quantified using the spectral image analysis method. The quantification was repeatedly on several places in the periphery of a culture dish to analyze the relative changes in the collagen contents.

Effect of treatment with PD98059, an inhibitor of the activation of extracellular-signal regulated kinase 1/2 (ERK1/2, also called p44/p42 MAPK), was studied on the absorbance of Sirius red in cells exposed to ELF-EMF. Figure 6A shows that PD 98059 significantly decreased the absorbance in all cells examined. However, the decreases caused by PD98059 in cells exposed to ELF-EMF (E) and in cells in the presence of IGF were both smaller than that in the control cells (C). We rearranged the same data and compared the effects of ELF-EMF exposure and IGF-I addition in the presence and absence of PD98059, separately, as shown in Fig. 6B. A slight but significant increase in the absorbance was observed in ELF-EMF exposure (E) without PD98059. In the presence of PD98059 the absorbance both on ELF-EMF exposure (E) and on IGF-I addition was significantly larger than that in control cells (C). The results indicated that treat-
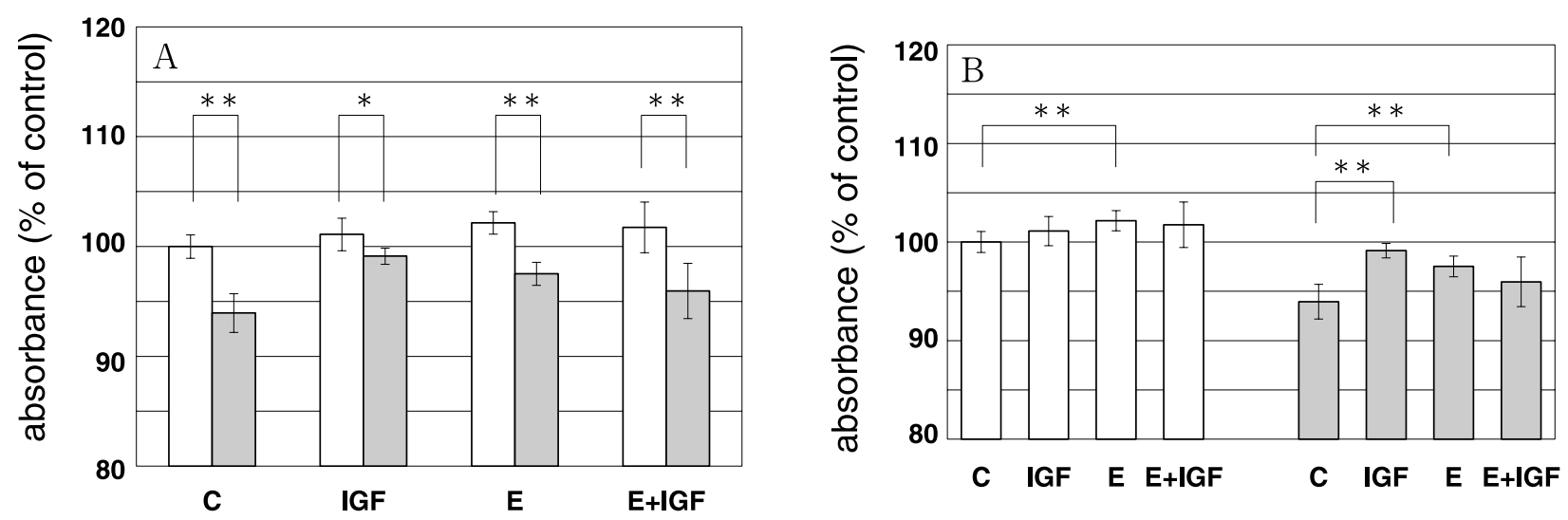

Fig. 6 The inhibitory effects of PD98059 on the absorbance of Sirius red-stained MC3T3-E1 cells exposed to ELF-EMF. Gray columns, addition of $10 \mu \mathrm{M}$ PD98059 for the last 3 days in a culture period of 14 days ; white columns, no addition. A : Effect of PD 98059 on cells exposed to ELF-EMF and on the cells in the presence of IGF-I. B : Effects of ELF-EMF exposure and addition of IGF-I on the PD98059-untreated cells (left) and PD98059-treated cells (right), plotted from the same data in A. In each culture dish 6 places at the periphery were selected at random for analysis. Symbols and the others as in Fig. 5 . 
ment with PD98059 did not inhibit the enhancing effect of ELF-EMF exposure on the absorbance.

Next, we examined the effect of an inhibitor of phosphatidylinositol 3- kinase (PI3K), LY294002, on the absorbance of Sirius red-stained cells. Treatment with LY294002 did not significantly influence the absorbance in the control cells ( $\mathrm{C}$ in Fig. 7A), whereas LY294002 significantly decreased the absorbance in the presence of IGF-I. On the other hand, the absorbance was significantly increased by ELF-EMF exposure when the inhibitor was present (E). In the absence of LY294002 both ELFEMF exposure and addition of IGF-I significantly increased the absorbance (Fig. 7B). In contrast, addition of IGF-I in the presence of LY294002 did not change the absorbance, but ELF-EMF expo- sure significantly increased the absorbance. The results showed that suppression of the PI3K pathway by LY294002 counteracted the increase effect in the collagen content caused by addition of IGFI, whereas the increase by ELF-EMF exposure was not inhibited but rather accelerated.

Finally, we examined the effect of an inhibitor of p38 mitogen-activated protein kinase (p38 MAPK), SB203580, on the absorbance of Sirius red-stained cells. On the cells unexposed to ELF-EMF, there were no significant effects of SB203580 on the absorbance with or without addition of IGF-I (Fig. 8 A). ELF-EMF exposure increased the absorbance in the absence of SB203580 (Fig. 8B, left), however, the inhibitor significantly decreased the absorbance in the exposed cells (Fig. 8A). In contrast, addition
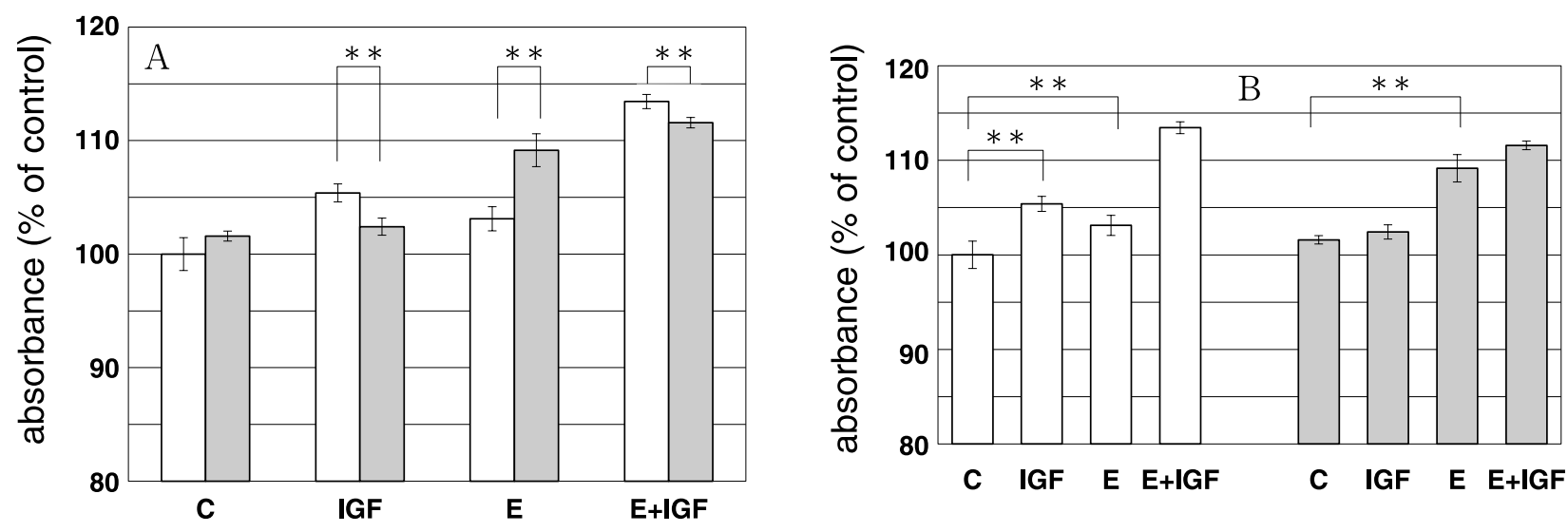

Fig. 7 The effects of LY294002 on the absorbance of Sirius red-stained MC3T3-E1 cells exposed to ELF-EMF. Gray columns, addition of $10 \mu \mathrm{M}$ LY294002 for the last 3 days in a culture period of 11 days ; white columns, no addition. A : Effect of LY294002 on the cells exposed to ELF-EMF and on the cells in the presence of IGF-I. B : Effects of ELF-EMF exposure and addition of IGF on the LY294002-untreated cells (left) and LY294002-treated cells (right). In each culture dish 4 places at the periphery were selected at random for analysis. Symbols and the others as in Fig. 6.
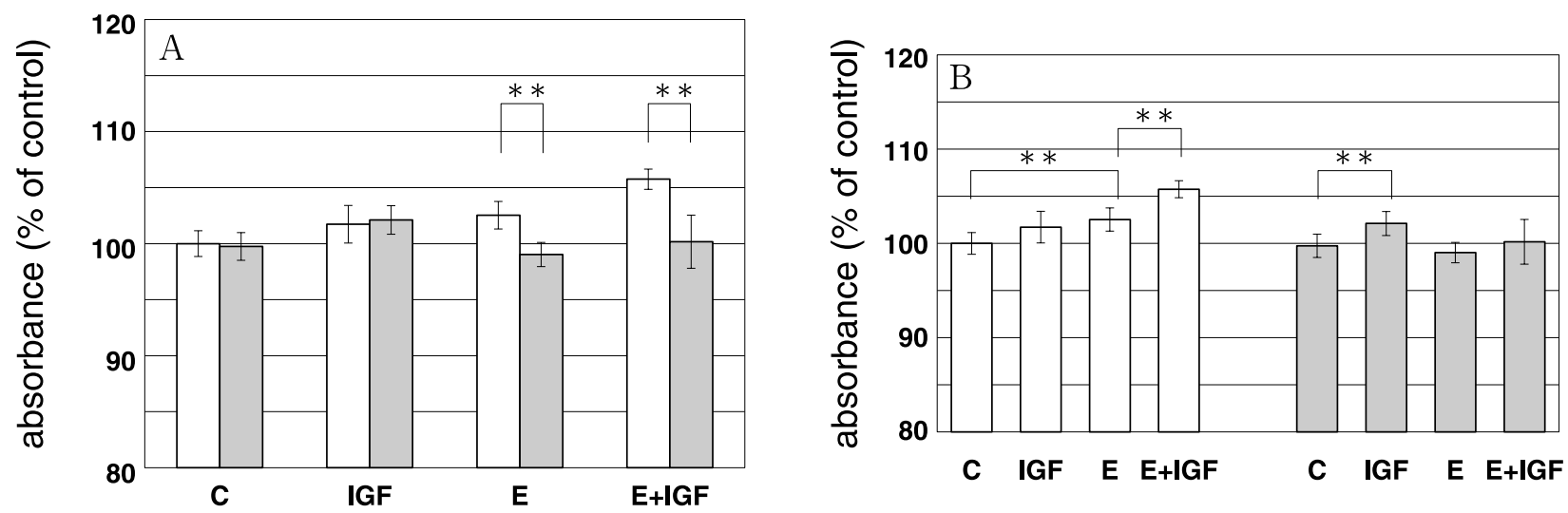

Fig. 8 The effect of SB203580 on the absorbance of Sirius red-stained MC3T3-E1 cells exposed to ELF-EMF. Gray columns, 10 $\mu \mathrm{M}$ SB203580 for the last 3 days in a culture period of 17 days; white columns, no addition. A : Effect of SB203580 on the cells exposed to ELF-EMF and on the cells in the presence of IGF-I. B : Effects of ELF-EMF-exposure and addition of IGF on the SB 203580-untreated cells (left) and SB203580-treated cells (right). In each culture dish 6 places at the periphery were selected at random for analysis. Symbols and the others as in Fig. 6. 
of IGF-I only increased significantly the absorbance when treated with the inhibitor (Fig. 8B, right). When SB203580 was present, the ELF-EMF exposure did not increase the absorbance. This implied that p38 MAPK pathway participated in the process to increase in the collagen content induced by ELF-EMF exposure.

Cells double-stained with Sirius red and Fast green were shown in Fig. 9. Red color corresponding to collagen seemed to be a little more distinct in the cells exposed to ELF-EMF and/or treated with IGF-I than the control cells. However, no marked change in cell morphology and orientation was detected.
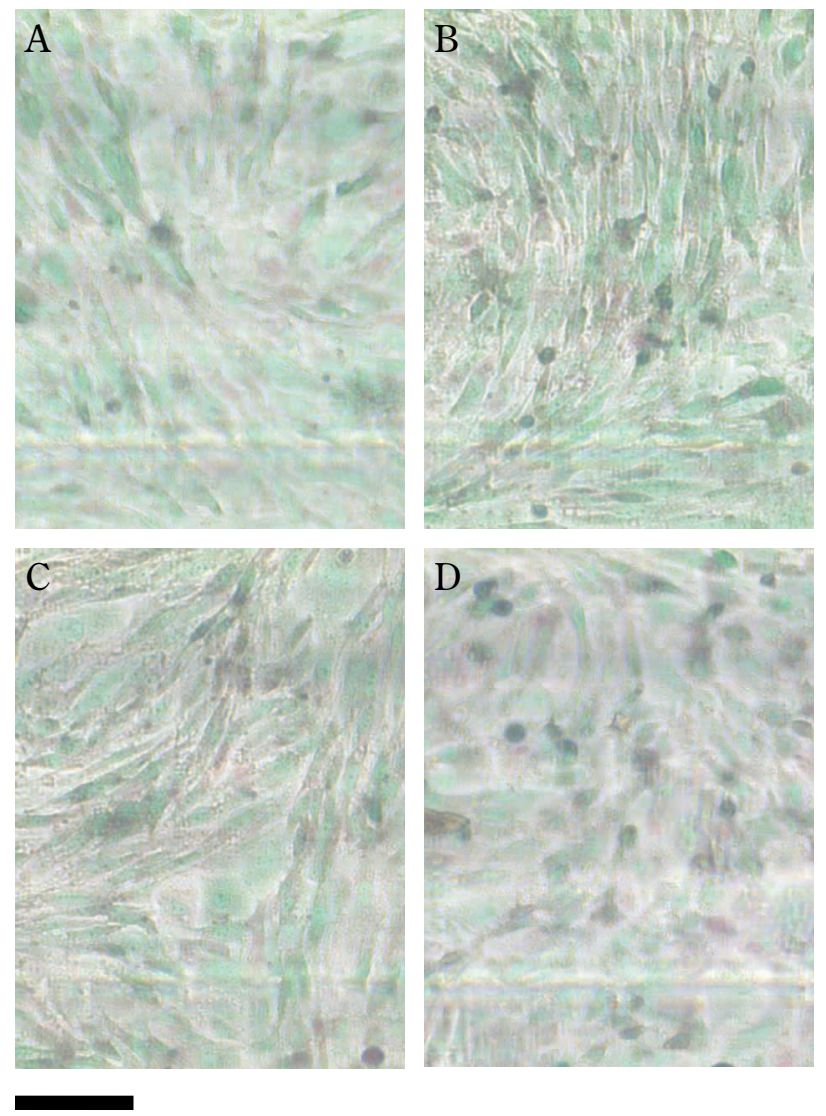

Fig. 9 MC3T3-E1 cells double-stained with Sirius red and Fast green. A : control ; B : addition of IGF-I ; C : exposure to ELF-EMF ; and D : exposure to ELF-EMF in the presence of IGF-I. Cells were exposed to ELF-EMF $(3 \mathrm{mT}, 60 \mathrm{~Hz})$ in the presence or the absence of IGF-I $(200 \mathrm{ng} / \mathrm{ml})$ for the last 3 days in a culture period of 18 days. The scale bar at the left bottom represents $100 \mu \mathrm{m}$ in length.

\section{DISCUSSION}

When we expose cells to ELF-EMF, there is a basic question to ask whether magnetic field or induced eddy current is effective to the cell function. In this study the magnetic flux density remained almost constant ( $3 \mathrm{mT})$ anywhere in the culture dish (Fig. 3). In contrast, the current density was small at around the center of the dish and it increased as the position approached to the periphery (equation [1]). The collagen content in cells, exposed to ELF-EMF, located at the periphery of the dish was significantly larger than that in cells located at around the center (Fig. 5A). The same tendency was observed in cells exposed to the ELF-EMF in the presence of IGF-I. This implied that eddy current is effective on the increase of collagen content. When the collagen content was large in the cells cultured for longer period of 21 days, the ELF-EMF exposure caused marked increase in the collagen content, however, the localization effect could not be observed (Fig. 5B), indicating that the small eddy current density at around the center would be large enough to stimulate the collagen production at the late stage of differentiation of the cells.

Is the eddy current induced inside the cells effective to stimulate the production of collagen in the cells? The density of eddy current calculated inside the cells would be at least three figures as small as it was in the medium at the periphery of the dish. Hence, the intracellular currents would be hard to influence cell functions. High impedance of cell membrane prevents penetration of the eddy current in the medium into cells across the membrane. Therefore, it is most reasonable to take that the current induced in the medium influence electric properties of certain structures on the cell surface (10), promoting the collagen synthesis.

Exposure to ELF-EMF $(60 \mathrm{~Hz}, 0.7 \mathrm{mT})$ has been reported to elicit the differentiation of chromaffin cells into sympathetic neuron-like cells (23). In human oral keratinocytes exposed to ELF-EMF (50 $\mathrm{Hz}, 2 \mathrm{mT}$ ), expression of differentiation marker protein was reported to increase, whereas expression of the receptor of epidermal growth factor decreased, indicating changes in keratocytes to higher differentiation level (24). On MC3T3-E1 cells ELFEMF exposure was reported to inhibit the gap junction communication, and the inhibition was not effective at the fully differentiated stage but effective at the early stage of differentiation (25). Exposure to pulsed electromagnetic fields was also reported to enhance cellular differentiation and to increase bone tissue-like formation in MC3T3-E1 cells (26). These studies suggest a possibility of facilitating collagen synthesis in MC3T3-E1 cells by exposure to EMF. Our results confirmed that exposure to an 
ELF-EMF $(60 \mathrm{~Hz}, 3 \mathrm{mT})$ enhanced collagen synthesis, and the effect was more evident in cells cultured long for 21 days than that in cells cultured for 14 days (Fig. 5).

Next, we tried to identify which members of the multiple signaling pathways were involved in the collagen synthesis induced by ELF-EMF exposure. Cells receive information from external environment by receptors on the surface membrane, and the message is transferred via the activation of various downstream signaling molecules. Among the most widely studied of these pathways are that involving the mitogen-activated protein kinase (MAPK) family, which regulates the proliferation and differentiation of osteoblasts.

In the MAPK family, ERK1/2 (also called p44/p 42 MAPK) plays a role in the signaling pathways in MC3T3-E1 cells. ERK1/2 activation is inhibited by PD98059 (16). Matrix mineralization by MC3T3E1 cells is enhanced by PD98059 treatment and is suppressed through ERK activation by plateletderived growth factor (27), indicating that ERK pathway participates on the process to suppress differentiation.

On the expression of alkaline phosphatase (ALP) activity in MC3T3-E1 cells the conflicting results were reported on the role of ERK1/2 pathway. Namely, PD98059 suppresses ALP activity induced by IGF-I in MC3T3-E1 cells (16), indicating that ERK1/2 pathway positively regulates the ALP activity. Conversely, PD98059 antagonizes the inhibitory action of transforming growth factor $\beta$ (TGF$\beta)$ on the ALP activity in the cells (21), indicating that ERK1/2 pathway negatively regulates ALP activity in the cells. No effect of PD98059 on ALP expression was also reported on primary culture of calvarial osteoblasts (28), but others reported the low ALP activity caused by PD98059 in MC3T3-E1 cells (29). We could not conclude whether ERK1/ 2 pathway participates positively or negatively on the expression of ALP activity in MC3T3-E1 cells.

Our results on collagen synthesis in MC3T3-E1 cells showed that ELF-EMF exposure increased in collagen content in all experiments (Figs. 6B, 7B, and $8 \mathrm{~B}$ ), however, the significant increase was observed only in an experiment with IGF-I addition (Fig. 7B). PD98059 decreased the collagen content in control and in the groups exposed to ELF-EMF and/or treated with IGF-I (Fig. 6A), suggesting a contribution of the collagen synthesis through the activation of ERK1/2 pathway.

On the other hand, the significant increase in collagen content was observed on the IGF-I addition and ELF-EMF exposure in the presence of PD98059 (Fig.6B). This implied that the stimulating effect of ELF-EMF exposure or addition of IGFI was, in part, counteracting on the suppressive action of PD98059 on ERK 1/2 pathway in collagen synthesis. However, the action was not additive between the IGF-I addition and ELF-EMF exposure. Of course, another explanation is possible such as the involvement of other pathways to enhance collagen synthesis by ELF-EMF exposure without the contribution of ERK $1 / 2$ pathway. We could not evaluate the exact role of the ERK $1 / 2$ pathway in collagen synthesis in MC3T3 cells. In fact, there is a report that TGF- $\beta$ induced production of type I collagen was not antagonized by PD 98059 (21).

Phosphatidylinositol 3-kinase (PI3K) plays a role in signaling in cell survival. In human osteoblastic cells PI3K pathway mediates IGF-I signals leading to mitogenesis and survival (19). The necessity of the pathway for suppression of apoptosis was pointed out in MC3T3-E1 cells using the inhibitor LY294002 (30). LY294002 was reported to inhibit ALP activity induced by IGF-I in the cells (31). We examined the effect of the inhibitor LY294002 on the collagen content, and found that the action of IGF-I to increase collagen was significantly suppressed (Fig. 7A). In contrast, the increase in collagen content induced by ELF-EMF exposure was not reduced but rather accelerated by the inhibition of PI3K pathway (Fig. 7B). This implied that collagen synthesis stimulated by IGF-I was, in part, carried out by the participation of PI3K pathway, however, that PI3K pathway may negatively regulate the collagen synthesis induced by ELFEMF exposure, and that the suppression of the pathway allows further collagen synthesis.

It has been reported that p38 MAPK is critical for the control of ALP expression in differentiating MC3T3-E1 cells (32). Inhibition of p38 MAPK significantly reduced the expression of differentiation markers such as ALP activity and mineral deposition (28), which indicates that p38 MAPK is necessary for differentiation of the cells. Parathyroid hormone (PTH) activated p38 MAPK in early differentiation of MC3T3-E1 cells, and the activation mediated PTH-induced stimulation of ALP activity (33). In bone morphogenic protein- 4 -stimulated osteocalcin synthesis, p38 MAPK has been pointed out to take part as a positive regulator, whereas ERK1/2 acts as a negative regulator in the synthe- 
sis in the cells (34). The involvement of p38 MAPK in the collagen expression was reported (15).

In the present study the increase in the collagen content in cells exposed to ELF-EMF was significantly counteracted by addition of the inhibitor of p38 MAPK, SB203580, whereas the increase caused by IGF-I addition was not affected by SB203580 (Fig. 8B). Thus, ELF-EMF exposure significantly stimulated collagen synthesis in differentiating cells, and the stimulation took place by participation of $p$ 38 MAPK pathway.

In conclusion, we could find that exposure to ELF-EMF increases significantly the collagen synthesis in osteoblast-like MC3T3-E1 cells. The effect seems to be caused by the eddy current in the culture medium induced by the ELF-EMF exposure. The collagen synthesis stimulated by the eddy current is carried out by participation of p38 MAPK pathway, and PI3K pathway may participate to suppress the collagen synthesis. The suppression of the PI3K pathway may allow the acceleration of collagen synthesis in the cells induced by the ELFEMF exposure.

\section{ACKNOWLEDGEMENTS}

We are deeply acknowledged to the late Dr. Hisao Yamaguchi for advice and discussion. We are indebted to Dr. Keiko Hosokawa, Dr. Hiroshi Miyamoto for discussion, and Dr. Katsuo Aizawa for advise on the microspectroscopy. We also thank Mr. Mitsuo Kitamura for technical assistance. This study was supported in part by Grant-in-Aid given to one of us from the Ministry of Education, Culture, Sports, Science and Technology of Japan (No. 19510032).

\section{REFERENCES}

1. Bassett CAL, Pawluk RJ, Becker RO : Effect of electrical currents on bone in vivo. Nature $204: 652-653,1964$

2. Basett CAL : Bioelectromagnetics in the service of medicine. In : Blanck M, ed. Electromagnetic Fields, Biological Interactions and Mechanisms. American Chemical Society, Washington DC, 1995, pp.261-275

3. Bassett CAL : Fundamental and practical aspects of therapeutic uses of pulsed electromagnetic fields (PEMFs). Crit Rev Biomed Eng
$17: 451-529,1989$

4. Miyamoto $H$, Yamaguchi $H$, Ikehara $T$, Kinouchi Y : Effects of electromagnetic fields on $\mathrm{K}^{+}\left(\mathrm{Rb}^{+}\right)$uptake by HeLa cells. In : Ueno $\mathrm{S}$, ed. Biological Effects of Magnetic and Electromagnetic Fields. Plenum Press, New York and London, 1996, pp.101-120

5. Ikehara T, Yamaguchi $\mathrm{H}$, Miyamoto $\mathrm{H}$ : Effects of electromagnetic fields on membrane ion transport of cultured cells. J Med Invest $45: 47-56,1998$

6. Sato K, Yamaguchi H, Miyamoto H, Kinouchi Y : Growth of cultured cells exposed to a nonhomogeneous static magnetic field generated by Sm-Co magnets. Biochim Biophys Acta 1136 : 231-238, 1992

7. Yamguchi H, Hosokawa K, Soda A, Miyamoto $\mathrm{H}$, Kinouchi Y : Effects of seven months' exposure to a static $0.2 \mathrm{~T}$ magnetic field on growth and glycolytic activity of human gingival fibroblasts. Biochim Biophys Acta 1156 : 302-306, 1993

8. Ikehara T, Park KH, Houchi H, Yamaguchi H, Hosokawa K, Shono M, Minakuchi K, Tamaki T, Kinouchi Y, Yoshizaki K, Miyamoto H : Effects of a time-varying strong magnetic field on transient increase in cytosolic free $\mathrm{Ca}^{2+}$ induced by bradykinin in cultured bovine adrenal chromaffin cells. FEBS Lett 435 : 229-232, 1998

9. Ikehara T, Yamaguchi H, Hosokawa K, Houchi H, Park KH, Minakuchi K, Kashimoto H, Kitamura M, Kinouchi Y, Yoshizaki K, Miyamoto $\mathrm{H}$ : Effects of time -varying strong magnetic field on transient increase in $\mathrm{Ca}^{2+}$ release induced by cytosolic $\mathrm{Ca}^{2+}$ in cultured pheochromocytoma cells. Biochim Biophys Acta $1724: 8-16,2005$

10. Yamaguchi H, Ikehara T, Hosokawa K, Soda A, Shono M, Miyamoto H, Kinouchi Y, Tasaka, $\mathrm{T}$ : Effects of time-varying electromagnetic fields on $\mathrm{K}^{+}\left(\mathrm{Rb}^{+}\right)$fluxes and surface charge of HeLa cells. Jpn J Physiol 42 : 929-943, 1992

11. Ikehara $T$, Yamaguchi $H$, Hosokawa $K$, Miyamoto H, Aizawa K: Effects of ELF magnetic field on membrane protein structure of living HeLa cells studied by Fourier transform infrared spectroscopy. Bioelectromagnetics 24 : 457-464, 2003

12. Lee JH, McLeod $\mathrm{KJ}$ : Morphologic responses of osteoblast-like cells in monolayer culture to ELF electromagnetic fields. Bioelectromag- 
netics $21: 129-136,2000$

13. Löschinger $\mathrm{M}$, Thumm $\mathrm{S}$, Hämmerle $\mathrm{H}$, Rodemann HP : Stimulation of protein kinase A activity and induced terminal differentiation of human skin fibroblasts in culture by lowfrequency electromagnetic fields. Toxicol Lett 96-97 : 369-376, 1998

14. Sudo H, Kodama H, Amagai Y, Yamamoto S, Kasai S : In vitro differentiation and calcification in a new clonal osteogenic cell line derived from newborn mouse calvaria. J Cell Biol $6: 191-198,1983$

15. Suzuki A, Guicheux J, Palmer G, Miura Y, Olso Y, Bonjour JP, Caverzasio J : Evidence for a role of $\mathrm{p}$-38 MAP kinase in expression of alkaline phosphatase during osteoblastic cell differentiation. Bone 30 : 91-98, 2002

16. Hanai $\mathrm{Y}$, Tokuda H, Ishisaki A, MatsushimaNishiwaki R, Nakamura N, Yoshida M, Takai $\mathrm{S}$, Ohta T, Kozawa $\mathrm{O}$ : Involvement of $\mathrm{p} 44 / \mathrm{p}$ 42 MAP kinase in insulin-like growth factor induced alkaline phosphatase activity in osteoblast-like-MC3T3-E1 cells. Mol Cell Endocrinol $251:$ :42-48, 2006

17. Chakravarthy MV, Abraha TW, Schwartz RJ, Fiorotto ML Booth FW : Insulin-like growth factor-I extends in vitro replicative life span of skeletal muscle satellite cells by enhancing $\mathrm{G}_{1} /$ $\mathrm{S}$ cell cycle progression via the activation of phosphatidylinositol 3'-kinase/Akt signaling pathway. J Biol Chem 275 : 35942-35952, 2000

18. Kuemmerle JF, Zhou H, Bowers JG : IGF-I stimulates human intestinal smooth muscle cell growth by regulation of $\mathrm{G} 1$ phase cell cycle proteins. Am J Physiol 286 : G412-G419, 2004

19. Grey A, Chen Q, Xu X, Callon K, Cornish J : Parallel phosphatidylinositol-3 kinase and p42/ 44 mitogen-activated protein kinase signaling pathways subserve the mitogenic and antiapoptotic actions of insulin-like growth factor I in osteoblastic cells. Endocrinology 144 : 48864893, 2003

20. Boguslawski G, Hale LV, Yu X-P, Miles RR, Onyia JE, Santerre RF, Chandrase KS : Activation of osteocalcin transcription involves interaction of protein kinase A- and protein kinase C-dependent pathways. J Biol Chem 275 : 999 1006,2000

21. Sowa H, Kaji H, Yamaguchi T, Sugimoto T, Chihara $\mathrm{K}$ : Activation of ERK1/2 and JNK by transforming growth factor $\beta$ negatively regulate Smad3-induced alkaline phosphatase ac- tivity and mineralization in mouse osteoblastic cells. J Biol Chem 277 : 36024-36031, 2002

22. Leon AL, Rojkind M : A simple micromethod for collagen and total protein determination in formalin-fixed paraffin-embedded sections. J Histochem Cytochem 33 : 737-743, 1985

23. Olivarez-Banuelos T, Navarro L, Gonzalez A, Drucker-Colin R: Differentiation of chromaffin cells elicited by ELF-MF modifies gene expression pattern. Cell Biol Intern 28 : 273-279, 2004

24. Manni V, Lisi A, Rieti S, Serafino A, Ledda M, Giuliani L, Sacco D, D'Emilia E, Grimaldi S : Low electromagnetic field $(50 \mathrm{~Hz})$ induces differentiation on primary human oral keratinocytes (HOK). Bioelectromagnetics 25 : 118126, 2004

25. Yamaguchi, DT, Huang J, Defang, MA, Wang PKC : Inhibition of gap junction intercellular communication by extremely low-frequency electromagnetic fields in osteoblast-like models is dependent on cell differentiation. J Cell Physiol 190 : 180-188, 2002.

26. Diniz P, Shomura K, Soejima K, and Ito G : Effects of pulsed electromagnetic field (PEMF) stimulation on bone tissue like formation are dependent on the maturation stages of the osteoblasts. Bioelectromagnetics $23: 398-405$, 2002

27. Kono S, Oshima Y, Hoshi K, Bonewald LF, Oda H, Nakamura K, Kawaguchi H, Tanaka $\mathrm{S}$ : Erk pathways negatively regulate matrix mineralization. Bone $40: 68-74,2007$

28. Hu Y, Chan E, Wang SX, Li B : Activation of $p$ 38 mitogen-activated kinase is required for osteoblast differentiation. Endocrinology 144 : 2068-2074, 2003

29. Khatiwala CB, Peyton SR, Metzke M, Putnam $\mathrm{AJ}$ : The regulation of osteogenesis by ECM rigidity in MC3T3-E1 cells requires MAP activation. J Cell Physiol 211 : 661-672, 2007

30. Golden LH, Insogna KL : The expanding role of PI3-kinase in bone. Bone 34 : 3-12, 2004

31. Noda T, Tokuda H, Yoshida M, Yasuda E, Hanai Y, Takai S, Kozawa O : Possible involvement of phosphatidylinositol 3-kinase/Akt pathway in insulin-like growth factor-induced alkaline phosphatase activity in osteoblasts. Horm Metab Res 37 : 270-274, 2005

32. Guicheux J, Lemonnier J, Ghayor C, Suzuki A, Palmer G, Caverzasio J : Activation of p38 mitogen-activated protein kinase and c-Jun- 
NH2-terminal kinase by BMP-2 and their implication in the stimulation of osteoblastic cell differentiation. J Bone Miner Res 18 : 20602068, 2003

33. Rey A, Manen D, Rizzoli R, Ferrari SL, Caverzasio $\mathrm{J}$ : Evidences for a role of p38 MAP kinase in the stimulation of alkaline phosphatase and matrix mineralizaion induced by parathyroid hormone in osteoblastic cells. Bone $41: 59-67,2007$

34. Kozawa O, Hatakeyama D, Uematsu T: Divergent regulation by $\mathrm{p} 44 / \mathrm{p} 42 \mathrm{MAP}$ and $\mathrm{p} 38$ kinase of bone morphogenetic protein-4-stimulated osteocalcin synthsis in osteoblasts. J Cell Biochem 84 : 583-589, 2002 